\title{
Active Power Sharing and Frequency Regulation in Droop-Free Control for Islanded Microgrids Under Electrical and Communication Failures
}

\author{
Carlos Xavier Rosero, Manel Velasco, Pau Martí, Antonio Camacho, Jaume Miret, Member, IEEE, \\ and Miguel Castilla
}

\begin{abstract}
The droop-free control for AC microgrids achieves active power sharing and frequency regulation by replacing the centralized secondary control and the primary-level droop mechanism by a cooperative distributed control strategy. This paper analyses the performance of the droop-free control when an islanded microgrid suffers electrical and communication failures. The microgrid is modeled by two connected graphs corresponding to the electrical and communication networks. The considered failures leads to partitioned electrical/communication subgraphs that co-exist within the microgrid. A closed-loop model integrating the power flow equations, the droop-free control, and the electrical and communication graphs is derived. The stability analysis identifies critical partitions where the microgrid is driven to the instability, and the steady-state analysis characterizes the equilibrium points when the partitioned microgrid remains stable. The paper also provides a control strategy that is able to avoid the instability scenario. Selected experimental results on a low-scale laboratory microgrid illustrate the impact that failures have in system performance and the ability of the proposed control for confining the microgrid operation into a safe region.
\end{abstract}

Index Terms-Microgrids, islanded mode, power sharing, frequency regulation, droop-free control, graph theory, partitioning.

\section{INTRODUCTION}

Microgrids (MGs) are small power systems integrating loads, sources and storage devices, that are interfaced with the electric network via power electronic inverters [1]. Their operation increasingly relies on the availability of communications [2]. The interdependency between the electrical and the communication networks that makes the problems of reliability and operation more complex than in the traditional grid has been treated in the literature (see a recent review in [3]).

Damage in electric lines or failures in the communication infrastructure negatively impact power systems [4]. The most severe electrical failures are power outages because they disrupt the electricity supply for an extended time resulting in the loss of critical services. Complementary, communication

This work was supported by the Ministry of Science, Innovation and Universities of Spain and by the European Regional Development Fund under project RTI2018-100732-B-C22.

C.X. Rosero, M. Velasco, P. Martí and A. Camacho are with the Automatic Control Department, Technical University of Catalonia, Pau Gargallo 5, 08028 Barcelona, Spain, email: cxrosero@utn.edu.ec, \{manel.velasco,pau.marti,antonio.camcho.santiago\}@upc.edu

J. Miret and M. Castilla are with the Electronic Engineering Department, Technical University of Catalonia, Av. Victor Balaguer s/n, 08800 Vilanova la Geltrú, Spain, email:\{jaume.miret,miquel.castilla\} @upc.edu failures and denial-of-service attacks paralyze data communications and difficult power management. The goal of reliable approaches to modern MG control and management is to ridethrough electrical and communication failures providing power quality graceful degradation but without causing loss of power supply nor serious damage to hardware assets, see e.g. [5][9] and references therein. However, none of the previous approaches has treated failures that generate MG partitions at the electrical and communication level.

This paper considers failures in islanded MGs controlled by physically distributed voltage source inverters (VSI) that exchange control data over a communication network. The control algorithm that is analyzed implements a subset of the droop-free control approach [10] for active power sharing and frequency regulation. The droop-free control approach is a distributed solution to satisfy both the secondary and the primary control level objectives of the standard MG hierarchical control scheme [11]. Together with the distributed frequency/active power droop-free control, a local voltage/reactive power droop control [12] is also considered in each VSI.

The focus of the paper is on failures in terms of damage in power links and/or loss of communication between nodes in such a way that the MG is partitioned. The MG is modeled by two connected graphs corresponding to the electrical and communication networks. In [10] it is studied the resiliency of the droop-free control to a single communication link failure when it does not alter the connectivity of the communication graph. This paper complements [10] by considering failures that leads to disconnected electrical/communication sub-graphs, named partitions, that co-exist within the MG. A closed-loop model integrating the two graph Laplacian matrices is built and analyzed using the zero eigenvalue analysis. This technique has been also used in the stability analysis of power systems of critical lines [13] and in the stability analysis for partitioned MGs controlled by consensus-based secondary control [14]. However, its application to the performance analysis of the droop-free control has not been reported.

The theoretical study permits identifying failures that lead to critical scenarios where voltage deviations and frequency fluctuations occur, and where the microgrid is driven to the instability. To overcome this problem, this paper presents a distributed control strategy based on a switched control policy at each VSI that ensures a graceful operation of the faulty system (partitioned MG) without requiring the disconnection of loads, strategy that for example would be taken when 
applying load shedding policies (e.g. [15]). The proposed solution is theoretically analyzed from a stability point of view, and implementation aspects are also discussed. Selected experimental results on a low-scale laboratory MG illustrate the impact of failures and the ability of the proposed control for confining the microgrid operation into a safe region.

The rest of the paper is structured as follows. Section II introduces the closed-loop model. Section III provides the partition analysis and Section IV presents the proposed switched control strategy. Section V illustrates the different results with selected experiments. Section VI concludes the paper.

\section{MODELING APPROACH}

The MG electrical network is a generic connected grid where loads are modeled by balanced three-phase constant impedances. Although future work will consider more rich configurations (e.g. non-linear and time-varying loads), keeping a simplified model helps gaining understanding and reaching results that will permit dealing with more complex MGs. A Kron reduction is performed which allows obtaining a lower dimensional dynamically-equivalent model described by ordinary differential equations [16]. The reduced network is modeled as a connected undirected graph $\mathcal{G}_{e}=\left\{\mathcal{N}_{e}, \mathcal{E}_{e}\right\}$ where the $n_{e}$ nodes $\mathcal{N}_{e}$ represent DGs interfaced with VSIs and edges $\mathcal{E}_{e} \subseteq \mathcal{N}_{e} \times \mathcal{N}_{e}$ represent the power lines. Nodes are characterized by a phase angle $\theta_{i}$ and a voltage amplitude $v_{i}$. Edges represent line admittances between nodes $i$ and $j$ as $y_{i j}=g_{i j}+\mathrm{j} b_{i j} \in \mathbb{C}^{+}$, where $g_{i j} \in \mathbb{R}^{+}$is the conductance and $b_{i j} \in \mathbb{R}^{+}$is the susceptance. The electrical network is represented by the symmetric bus admittance matrix $Y \in \mathbb{C}^{n_{e}} \times \mathbb{C}^{n_{e}}$, where the off-diagonal elements are $Y_{i j}=Y_{j i}=-y_{i j}$ for each edge $\{i, j\} \in \mathcal{E}_{e}$, and the diagonal elements are given by $Y_{i i}=\sum_{i=1}^{n_{e}} y_{i j}$. It is assumed that the reduced $\mathrm{MG}$ is connected.

For balanced AC microgrids, the active power injected by each $i^{\text {th }}$ node of the $n$-node MG is described as

$$
p_{i}(t)=v^{2} \sum_{j=1}^{n} g_{i j}+v^{2} \sum_{j=1}^{n} b_{i j}\left(\theta_{i}(t)-\theta_{j}(t)\right)
$$

assuming that nodes phase angles are similar while voltages are constant and equal, as often assumed in power systems modeling, e.g. [17], and also in $\mathrm{MG}$ modeling, e.g. [18]. By considering the matrix $G \in \mathbb{R}^{n \times n}$ formed by the line conductances whose entries are given by $G_{i j}=g_{i j}$, denoting the set of phase angles by $\Theta(t)=\left[\theta_{1}(t) \cdots \theta_{n}(t)\right]^{T}$, and the set of active powers by $P(t)=\left[p_{1}(t) \cdots p_{n}(t)\right]^{T}$, the active power of the Kron-reduced network (1) becomes

$$
P(t)=v^{2} G \mathbf{1}_{n \times 1}+v^{2} B \Theta(t)
$$

where $\mathbf{1}_{n \times 1} \in \mathbb{R}^{n \times 1}$ denotes a vector of ones, and $B \in \mathbb{R}^{n \times n}$ is the Laplacian matrix of the power system given by

$$
B=\left[\begin{array}{cccc}
\sum_{\substack{j=1 \\
j \neq 1}}^{n} b_{1 j} & -b_{12} & \cdots & -b_{1 n} \\
-b_{21} & \sum_{\substack{j=1 \\
j \neq 2}}^{n} b_{2 j} & \cdots & -b_{2 n} \\
\vdots & \vdots & \cdots & \vdots \\
-b_{n 1} & -b_{n 2} & \cdots & \sum_{\substack{j=1 \\
j \neq n}}^{n} b_{n j}
\end{array}\right]
$$

and formed by the line susceptances.

The goal of the droop-free control is to achieve power sharing while having the frequency at the desired set-point. Active power sharing (i.e., the power of the inverters in steadystate must be equal while guaranteeing the supply of the load) can be formulated as $p_{i}(\infty)=\frac{P_{T}}{n}$ where $p_{i}(\infty)$ is the active power provided by each inverter in steady-state and $n$ is the number of VSIs of the MG. Frequency regulation in steady-state in the MG can be formulated as $\omega_{i}(\infty)=\omega_{0}$ where $\omega_{i}(\infty)$ is the local frequency of each inverter in steadystate. Each node $i \in \mathcal{N}_{e}$ is modeled as a control algorithm implemented at each VSI driven by

$$
\begin{aligned}
\omega_{i}(t) & =\omega_{0 i}+\delta_{i}(t)+\varphi_{i}(t) \\
v_{i}(t) & =v_{0 i}-n q_{i}(t) .
\end{aligned}
$$

In the droop-free control (4), each node frequency $\omega_{i}(t)$ is given by the set-point frequency $\omega_{0 i}$ and a correction term $\delta_{i}(t)$ that calculates the neighborhood active loading mismatch

$$
\delta_{i}(t)=c\left(\left(\sum_{j=1}^{n_{i}} a_{i j} p_{j}(t)\right)-n_{i} p_{i}(t)\right)
$$

where the coupling gain $c>0$ is a design parameter and the coefficients $a_{i j}$ determine the availability of communication between nodes $i$ and $j$, thus indicating the set of nodes $n_{i}$ that exchange control data. Typically, $n_{i}$ is the number of nodes that participate in the communication scheme. The droop-free control (4) is complemented with a perturbation term $\varphi_{i}(t)$ modeling bounded uncertainties such as measurement errors or disturbances. Although not explicitly formalized in (4), and for fairness in all the implementations, the droop-free control used in the experimental set-up will be implemented with the same output virtual impedance control used in the voltage droop control [19]. However, it is omitted in the model because its inclusion would not alter the theoretical results. The voltage droop control (5) for local reactive power $q_{i}(t)$ and voltage $v_{i}(t)$ regulation, where $v_{0 i}$ is the nominal voltage amplitude and $n$ the proportional control gain, is always enabled for voltage regulation, but it is not covered by the analysis.

The communication network can also be represented by a connected undirected graph $\mathcal{G}_{c}=\left\{\mathcal{N}_{c}, \mathcal{E}_{c}\right\}$ where the $n_{c}$ nodes $\mathcal{N}_{c}$ represent VSIs that implement (4)-(6), and edges $\mathcal{E}_{c} \subseteq$ $\mathcal{N}_{c} \times \mathcal{N}_{c}$ represent communication links. Parameters $a_{i j}$ in (6) form the adjacency matrix of $\mathcal{G}_{c}$ such that $a_{i j}=a_{j i}=1$ if nodes $i$ and $j$ can exchange their information and $a_{i j}=$ 0 otherwise. It is considered that nodes in the electrical and communication graph are the same, i.e. $\mathcal{N}_{e} \equiv \mathcal{N}_{c}$, hence $n_{e}=$ $n_{c}=n$, which is the habitual situation in MGs, e.g. [20].

By denoting the set of VSI local frequencies by $\Omega(t)=$ $\left[\omega_{1}(t) \cdots \omega_{n}(t)\right]^{T}$, the set of desired frequencies by $\Omega_{0}=$ $\left[\omega_{01} \cdots \omega_{0 n}\right]^{T}$, the set of active powers by $P(t)=$ $\left[p_{1}(t) \cdots p_{n}(t)\right]^{T}$, and the set of perturbations by $\Phi(t)=$ $\left[\varphi_{1}(t) \cdots \varphi_{n}(t)\right]^{T}$ the per-node control algorithm given in (4)-(6) can be compactly written for the entire MG as

$$
\Omega(t)=\Omega_{0}-c L P(t)+\Phi(t)
$$


where $L \in \mathbb{R}^{n \times n}$ is the Laplacian matrix of the communication graph $\mathcal{G}_{c}$ given by

$$
L=\left[\begin{array}{cccc}
\sum_{\substack{j=1 \\
j \neq 1}}^{n} a_{1 j} & -a_{12} & \cdots & -a_{1 n} \\
-a_{21} & \sum_{\substack{j=1 \\
j \neq 2}}^{n} a_{2 j} & \cdots & -a_{2 n} \\
\vdots & \vdots & \cdots & \vdots \\
-a_{n 1} & -a_{n 2} & \cdots & \sum_{\substack{j=1 \\
j \neq n}}^{n} a_{n j}
\end{array}\right]
$$

where $a_{i j}$ are the entries of the adjacency matrix of $\mathcal{G}_{c}$. The compact form of (5) that would accompany (7) is omitted because it is not further used.

The goal of the control (7) is shaping the active power and frequency dynamics. The active power dynamics can be obtained by computing the derivative of (2) that leads to

$$
\dot{P}(t)=v^{2} B \dot{\Theta}(t) .
$$

By being $\omega_{i}=\dot{\theta}_{i}$, the active power variation (9) is written as

$$
\dot{P}(t)=v^{2} B \Omega(t) .
$$

By substituing (7) into (10), the closed-loop dynamics can be written as

$$
\dot{P}(t)=S P(t)+U \Omega_{0}+R \Phi(t)
$$

where the closed-loop system matrix $S \in \mathbb{R}^{n \times n}$, input matrix $U \in \mathbb{R}^{n \times n}$, and perturbation matrix $R \in \mathbb{R}^{n \times n}$ are

$$
\begin{aligned}
S & =\left[-v^{2} c B L\right] \\
U & =\left[v^{2} B\right] \\
R & =\left[v^{2} B\right] .
\end{aligned}
$$

For completeness, the simple closed-loop dynamics (11) are complemented by the filter dynamics of the active power of each VSI, which are all embedded in a single state-space form named filter and given by

$$
\begin{aligned}
\dot{\hat{P}}(t) & =A^{F} \hat{P}(t)+B^{F} P(t) \\
\bar{P}(t) & =C^{F} \hat{P}(t)
\end{aligned}
$$

where the input of the filter is the measured $P(t)$, the filter internal state representing the filtered active power is denoted by $\hat{P}(t)$, and the filter output is the filtered active power $\bar{P}(t)$, which will be fed into (11). In (15)-(16), the system matrix $A^{F} \in \mathbb{R}^{(n m) \times(n m)}$, the input matrix $B^{F} \in \mathbb{R}^{(n m) \times n}$ and the output matrix $C^{F} \in \mathbb{R}^{n \times(n m)}$ are block diagonal matrices characterizing the $n$ filters of order $m$, whose particular structure is given by their diagonal elements $a_{i}^{F} \in \mathbb{R}^{m \times m}$, $b_{i}^{F} \in \mathbb{R}^{m \times 1}, c_{i}^{F} \in \mathbb{R}^{1 \times m}, i=1 \ldots, n$. It is assumed that $A^{F}$ is non-singular (i.e. its inverse exists), and that all filters are equally designed, which implies that the $n$ filters are characterized by the same static gain $\gamma \in \mathbb{R}$. Note that $P(t), \bar{P}(t) \in \mathbb{R}^{n \times 1}$ while $\hat{P}(t) \in \mathbb{R}^{(n m) \times 1}$.

By replacing $P(t)$ by $\bar{P}(t)$ in (11), and considering also (15)-(16), the complete closed-loop dynamics are given by

$$
\left[\begin{array}{c}
\dot{P}(t) \\
\dot{\hat{P}}(t)
\end{array}\right]=S^{F}\left[\begin{array}{c}
P(t) \\
\hat{P}(t)
\end{array}\right]+U^{F} \Omega_{0}+R^{F} \Phi(t)
$$

where

$$
\begin{aligned}
S^{F} & =\left[\begin{array}{cc}
0_{n \times n} & S C^{F} \\
B^{F} & A^{F}
\end{array}\right] \\
U^{F} & =\left[\begin{array}{c}
U \\
0_{(n m) \times n}
\end{array}\right] \\
R^{F} & =\left[\begin{array}{c}
R \\
0_{(n m) \times n}
\end{array}\right]
\end{aligned}
$$

where $0_{n \times n}$ or $0_{(n m) \times n}$ are matrices of $n \times n$ or $(n m) \times n$ zeros, respectivelly.

For the partition analysis it is assumed that the droopfree control gain $c$ in (6) has been designed such that both control goals, active power sharing and frequency regulation, can be always reached. This holds for the scenario previous to a partition and for the new scenario appearing after a partition. It is also assumed that after any electrical partition, sub-MGs remain balanced and each sub-MG meets the local load demands with the local generation units. Otherwise, a cascading failure would occur leading to a collapse [21], [22].

\section{PARTITION ANALYSIS}

This section covers stability and steady-state analysis. The stability analysis identifies which type of partition may lead to MG instability. This is achieved by studying the effect that a single partition occurring in one of two graphs has in the MG dynamics. The steady-state analysis indicates how to compute the equilibrium points for stable dynamics.

\section{A. Stability Analysis}

The stability of the droop-free closed-loop system (17) subject to partitions is based on structural properties of the involved Laplacian matrices using the zero eigenvalue analysis. In particular, it is derived by studying how new 0 eigenvalues appearing in the Laplacian matrices, $B(3)$ and $L(8)$, that are caused by partitions, can alter the closed-loop dynamics. And this is achieved by analyzing rank properties of the involved system $S^{F}$ (18) and input $U^{F}(19)$ matrices of the complete closed-loop dynamics (17). Note that $R^{F}$ (20) is equal to $U^{R}$ (19), and then it can be omitted in the rank analysis.

By noting that the static gain of the filter (15)-(16) is $C^{F}\left(-A^{F}\right)^{-1} B^{F}=\gamma \mathcal{I}_{n x n}$ (being $\mathcal{I}_{n x n} \in \mathbb{R}^{n \times n}$ the identity matrix), for the rank of the system matrix $S^{F}$ (18) it holds [23]

$$
\begin{aligned}
\operatorname{rank}\left(S^{F}\right)= & \operatorname{rank}\left(A^{F}\right) \\
& +\operatorname{rank}\left(0_{n \times n}+S C^{F}\left(A^{F}\right)^{-1} B^{F}\right) \\
& \operatorname{rank}\left(A^{F}\right)+\operatorname{rank}(-S) .
\end{aligned}
$$

In addition, by noting that the filter system matrix $A^{F}$ does not depend on the Laplacian matrices and has a constant rank regardless of the considered partitions, the study of rank of $S^{F}$ (18) simplifies into only studying the rank of $S$ (12). A similar conclusion applies to the rank of the input matrix $U^{F}$ (19), which only depends on the rank of $U$ (13). In other words, the analysis of the structural properties of the complete dynamics (17) subject to partitions can be performed in the simple dynamics (11) and then generalized. 
Since the starting point for the analysis is that both graphs are connected, it holds that their Laplacian matrices have a single 0 eigenvalue [24], which implies that $\operatorname{rank}(B)=$ $\operatorname{rank}(L)=n-1$. Whenever a partition occurs, the multiplicity of the 0 eigenvalue of the Laplacian matrices indicate the number of resulting disconnected components [24]. Hence, the location of the point of failure that leads to disconnected sub-graphs is not relevant. However, the role of the new 0 eigenvalue in the Laplacian matrices becomes crucial.

The study of the rank properties of the simple closedloop dynamics (11) must be complemented with the system restriction imposed by the power balance equation,

$$
\forall t, \quad \sum_{i=1}^{n} p_{i}(t)=P_{T}
$$

that indicates that for a given load, the total power $P_{T}$ (that includes the power losses) that is injected by the MG nodes is always the same. Its derivative is given by

$$
\sum_{i=1}^{n} \dot{p}_{i}(t)=0 .
$$

This means that one of the $\dot{p}_{i}(t)$ is linearly dependent of the others $\dot{p}_{j}(t), j=1, \ldots, n, j \neq i$, that is, 1 of the $n$ equations of (11) is linear dependent of the others. Looking at matrices $S$ (12) and $U$ (13), this dependency implies that $\operatorname{rank}(S)=$ $\operatorname{rank}(S \mid U) \leq n-1$ and also implies that at least $S$ has one eigenvalue at zero that does not act as an integrator for the closed-loop dynamics, thus not having destabilizing effects. Note also that $\operatorname{rank}(S \mid R) \leq n-1$, that is, the same structural property that exhibits $U$ applies to $R$ in (11).

By noting that any 0 eigenvalue implies that a matrix looses rank by one, and using the rank inequality [23]

$$
\operatorname{rank}(B L) \leq \min (\operatorname{rank}(B), \operatorname{rank}(L))
$$

the following properties can be concluded. When looking at the additional 0 eigenvalue appearing in the communication Laplacian matrix $L$ caused by a communication partition, it can be observed that the system matrix $S$ (12) depends on the product of both Laplacian matrices, $B L$, while the input matrix $U$ (13) does not depend on $L$. Hence, this additional 0 eigenvalue becomes an integrator for the closed-loop dynamics (11) because if $L$ looses rank, then matrix $S$ loses rank due to (24) while matrix $S \mid U$ does not, thus maintaining the original set of $n-1$ linear independent equations. However, this is not the case for the additional 0 eigenvalue appearing in the electrical Laplacian matrix $B$ caused by an electrical partition because both $S$ and $U$ depend on $B$. In this case, the additional 0 eigenvalue does not become an integrator for the closed-loop dynamics (11) because both matrices $S$ and $S \mid U$ loose rank if $B$ looses rank, thus decrementing the set of linear independent equations. And the same conclusions apply for the complete closed-loop dynamics (17).

Whenever a communication partition occurs, and reminding that the closed-loop system is multiple-input/multiple-output, the additional 0 eigenvalue becomes an integrator of each input/output relation (from input $\omega_{0 i}$ to any of the outputs).
Then, the system operation corresponds to $n$ integrators working in parallel, which puts the MG into risk [25], and implies unstable dynamics for the perturbed system (17). Whenever an electrical partition occurs, the additional 0 eigenvalue does not have the destabilizing effects as in the case of the communication partition. However, power flows can not be transferred between the isolated MGs and cascading failures could occur if each sub-microgrid supply-demand would not be able to reach the equilibrium. However, the adopted model assumes that the MG capacity has been dimensioned and control gains have been designed such that this equilibrium can be always reached. Hence, after the electrical partition, each MG will reach different steady-state equilibrium points.

\section{B. Steady-State Analysis}

The $n$ equilibrium points of the stable MG driven by the droop-free control depend on solving the active power closedloop dynamics (17) in steady state,

$$
\begin{aligned}
{\left[\begin{array}{l}
0 \\
0
\end{array}\right]=} & {\left[\begin{array}{cc}
0_{n \times n} & S C^{F} \\
B^{F} & A^{F}
\end{array}\right]\left[\begin{array}{c}
P(t) \\
\hat{P}(t)
\end{array}\right] } \\
& +\left[\begin{array}{c}
U \\
0_{(n m) \times n}
\end{array}\right] \Omega_{0}+\left[\begin{array}{c}
R \\
0_{(n m) \times n}
\end{array}\right] \Phi(t) .
\end{aligned}
$$

By using (12)-(14), eq. (25) can be written as

$$
\begin{aligned}
& 0=-v^{2} c B L C^{F} \hat{P}(t)+v^{2} B \Omega_{0}+v^{2} B \Phi(t) \\
& 0=B^{F} P(t)+A^{F} \hat{P}(t) .
\end{aligned}
$$

By noting that the set-point frequency for all VSIs is the same, $\omega_{0 i}=2 \pi 60 \mathrm{rad} / \mathrm{s}$, it holds that $B \Omega_{0}=0$ (because $B$ is a Laplacian matrix). In addition, by isolating $\hat{P}(t)$ in (27) and substituting it in (26), and reminding that static gain of the filter (15)-(16) is $C^{F}\left(-A^{F}\right)^{-1} B^{F}=\gamma \mathcal{I}_{n x n}$, equality (26) reduces to

$$
c B L \gamma \mathcal{I}_{n x n} P(t)=B \Phi(t)
$$

which is a system of $n-1$ equations due to the fact the Laplacian matrices (and in particular the electrical one, $B$ ) have at least a 0 eigenvalue (when no partitions occur). The missing equation for being able to find the $n$ equilibrium points was given by the MG power balance equation (22) and refers to the fact that the total injected power must meet the MG power demand. For each new electrical partition, a new 0 eigenvalue appears in $B$, and the number of equations in (28) is reduced by one while equation (22) is split into two equations, that is, increasing by one. The later is due to the fact that each new partition will obey a particular power balance, that is, it will have an equation specifying that the total injected power in that partition must meet that partition power demand. Hence, always $n$ equations will be available.

The generalization of the power balance equation (22) for a MG composed by $j$ partitions, each with a number of $k_{j}$ inverters meeting the partition power demand $P_{T j}$ is

$$
\left[\begin{array}{c}
g_{1} \\
g_{2} \\
\vdots \\
g_{j}
\end{array}\right]\left[\begin{array}{c}
p_{1}(t) \\
p_{2}(t) \\
p_{3}(t) \\
\vdots \\
p_{n}(t)
\end{array}\right]=\left[\begin{array}{c}
P_{T 1}(t) \\
P_{T 2}(t) \\
\vdots \\
P_{T j}(t)
\end{array}\right]
$$


where $g_{j} \in \mathbb{R}^{n}$ are linear independent vectors whose elements are 0 or 1 , and contain as many 1 s as VSIs are included in the $j$-partition. Eq. (29) can be compactly written as

$$
\mathcal{G P}(t)=\mathcal{P}_{T}(t) .
$$

The new power balance equation (30) together with (28) constitute a system of $n$ equations that can be written as

$$
\left[\begin{array}{c}
c B L \gamma \mathcal{I}_{n x n} \\
\mathcal{G}
\end{array}\right] P(t)=\left[\begin{array}{l}
B \Phi(t) \\
\mathcal{P}_{T}(t)
\end{array}\right]
$$

and whose finite solution for the case of stable dynamics provides the $n$ active power equilibrium points (characterized by the gain $\gamma$ of the active power) while the $n$ frequency equilibrium points can be then obtained using (7).

\section{PRoposed CONTROL STRATEGY}

The previous analysis has identified the communication partition as a source of MG instability. This section presents a control strategy thats avoids this problem by switching between droop-free and standard frequency droop control [12] whenever a communication partition is detected. Next subsections present the switched control strategy, the stability analysis, and implementation details. The steady-state analysis is not included because when droop-free control applies, the preceding analysis given in sub-section III-B holds, and when only frequency droop control applies, the steady-state behavior is well documented [12].

\section{A. Switched Control Algorithm}

The algorithm is as follows. In normal operation, distributed droop-free control applies thus guaranteeing active power sharing and frequency regulation. When a communication failure occurs, the droop-free control is disabled to avoid the instability scenario and frequency droop control is activated at each VSI. Hence, active power sharing can still be guaranteed while an unavoidable frequency deviation appears. Whenever the communication is re-established, frequency droop is disabled and droop-free control is restored to also regulate frequency. Both droop-free control and frequency droop control are executed together with the voltage droop control (5).

To enable the switched control algorithm, equation (4) written in terms of the filtered active power $\bar{p}_{i}(t)$ given by (15)-(16) is complemented with the frequency droop control method [12] in such a way that the application of frequency droop or droop-free control policies obeys the following equation

$$
\omega_{i}(t)=\omega_{0 i}-(1-\alpha) m \bar{p}_{i}(t)+\alpha \delta_{i}(t)+\varphi_{i}(t)
$$

where $\alpha \in\{0,1\}$ is a switching coefficient that determines its application. When $\alpha=1$, that is, in standard operation, the original droop-free control (4) is applied, where the correction term $\delta_{i}(t)$ is given in (6) where $p_{i}$ is replaced by $\bar{p}_{i}$. However, when $\alpha=0$, that is, in the occurrence of a communication partition, the frequency droop control characterized by droop gain $m>0$ is applied. And recall that in both scenarios, voltage droop control (5) always applies. In vector form, the switched control policy (32) can be rewritten as

$$
\Omega(t)=\Omega_{0}+\left(\left\{\begin{array}{cc}
-c L \bar{P}(t) & \text { if } \alpha=1 \\
-m \mathcal{I}_{n x n} \bar{P}(t) & \text { if } \alpha=0
\end{array}\right)+\Phi(t) .\right.
$$

The logics that determine the value of the switching coefficient $\alpha$ are provided in the implementation details sub-section IV-C.

\section{B. Stability Analysis of the Switched System}

For the stability analysis of the closed-loop system, it is noted that the application of the control law (33) together with the active power filter (15)-(16) leads to a switched linear system in which the two individual sub-systems are linear. The analysis is split into two steps. First, the stability of the switched system without considering the active power filtering is considered. Second, the inclusion of the filters is considered, leading to a switched system whose stability will depend on the specific filters.

1) Without Filtering: By substituting the proposed control (33) (changing the filtered active power $\bar{P}(t)$ by just $P(t)$ ) into (10), the active power dynamics can be written as

$$
\dot{P}(t)=S_{\alpha} P(t)+U \Omega_{0}+R \Phi(t)
$$

where the input matrix $U \in \mathbb{R}^{n \times n}$ and perturbation matrix $R \in \mathbb{R}^{n \times n}$ remain as defined in (13) and (14), respectively, but the closed-loop matrix $S_{\alpha} \in \mathbb{R}^{n \times n}$ (that replaces the original one $S$ given in (12)) is

$$
S_{\alpha}= \begin{cases}S_{1}=\left[-v^{2} c B L\right] & \text { if } \alpha=1 \rightarrow \text { droop-free } \\ S_{2}=\left[-v^{2} m B \mathcal{I}_{n x n}\right] & \text { if } \alpha=0 \rightarrow \text { freq. droop }\end{cases}
$$

It is well known that the stability of the individual subsystems does not guarantee the stability of the switched system. However, if the individual sub-systems share a common quadratic Lyapunov function with a negative semidefinite timederivative, then the switched system is stable for any arbitrary switching sequence [26]. Let

$$
V(P)=\frac{1}{2} P^{T} P
$$

be a candidate Lyapunov function for both sub-systems. Its derivative along the trajectories given by the droop-free control characterized by $S_{1}$ in (35) can be written as

$$
\dot{V}=P^{T} \dot{P}=P^{T}\left(-v^{2} c B L P\right)=-P^{T} v^{2} c B L P \leq 0 .
$$

The last inequality holds because it is observed first that both Laplacian matrices $B$ and $L$ are symmetric PSD (positive semidefinite) and their product is also symmetric. Therefore, it can be stated that $B L$ is PSD [27], that is $B L \geq 0$. And noting also that $c>0$ in (6), it can be concluded that $v^{2} c B L \geq 0$ and then the inequality in (37) holds. A similar analysis applied to the frequency droop control characterized by $S_{2}$ (35) leads to

$$
\dot{V}=P^{T} \dot{P}=P^{T}\left(-v^{2} m B \mathcal{I}_{n x n} P\right)=-P^{T} v^{2} m B P \leq 0
$$

As before, the last inequality holds because the Laplacian matrix $B$ is positive semidefinite, the product $v^{2} m B \geq 0$ (recall that $m>0$ in (32)) and then the inequality (38) holds. Hence, since both linear sub-systems characterized by the 


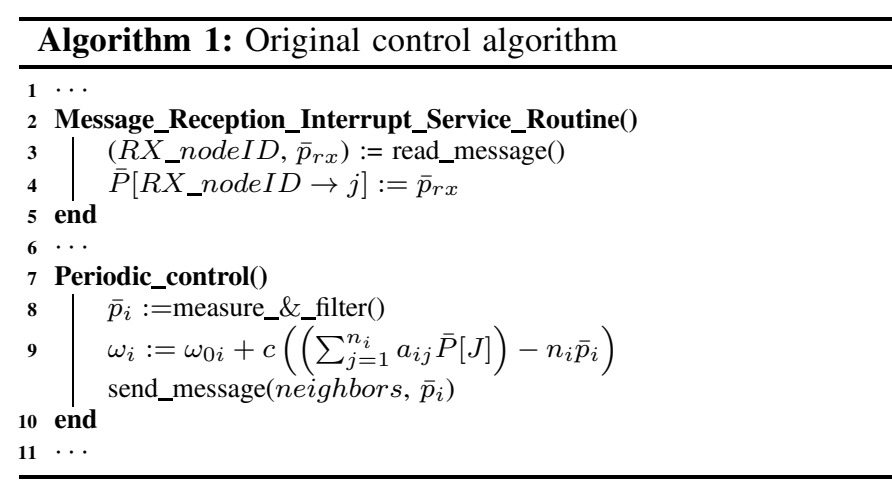

closed-loop matrices given in (35) share a common Lyapunov function (36), the switched system (34) without considering the filters of the active power is stable.

2) With Filtering: Considering the complete closed-loop dynamics (17)-(20) that includes the filters of the active power (15)-(16), together with the proposed control (33), the following switched system

$$
\left[\begin{array}{c}
\dot{P}(t) \\
\dot{\hat{P}}(t)
\end{array}\right]=S_{\alpha}^{F}\left[\begin{array}{c}
P(t) \\
\hat{P}(t)
\end{array}\right]+U^{F} \Omega_{0}+R^{F} \Phi(t)
$$

where

$$
S_{\alpha}^{F}=\left[\begin{array}{cc}
0_{n \times n} & S_{\alpha} C^{F} \\
B^{F} & A^{F}
\end{array}\right]
$$

characterized by $S_{\alpha}$ (35) is obtained. The stability of the switched system (39)-(40) can be assessed using linear matrix inequalities. By noting that the contribution of the input term $\left(U^{F} \Omega_{0}\right)$ in (39) is zero (due to the fact that if all set-point frequency are the same, it holds that $B \Omega_{0}=0$ ), by recalling the boundedness of the perturbations $\Phi(t)$, system (39)-(40) will be stable if it exists a positive definite matrix $M \in$ $\mathbb{R}^{(n+n m) \times(n+n m)}, M^{T}=M$, such that

$$
\begin{aligned}
& \left(S_{\alpha, 1}^{F}\right)^{T} M+M S_{\alpha, 1}^{F}<0 \\
& \left(S_{\alpha, 2}^{F}\right)^{T} M+M S_{\alpha, 2}^{F}<0
\end{aligned}
$$

with

$$
S_{\alpha, 1}^{F}=\left[\begin{array}{cc}
0_{n \times n} & S_{1} C^{F} \\
B^{F} & A^{F}
\end{array}\right], S_{\alpha, 2}^{F}=\left[\begin{array}{cc}
0_{n \times n} & S_{2} C^{F} \\
B^{F} & A^{F}
\end{array}\right]
$$

where $S_{1}$ and $S_{2}$ were given in (35). Hence, the stability will depend on the specific filters included in the system model.

\section{Implementation Details}

The implementation of the proposed control implies altering the basic code to be executed at each VSI. Algorithm 1 illustrates the standard operation given by (4) while Algorithm 2 presents the modifications that are required to implement (32). Each algorithm contains two parts. The first one is the interrupt service routine (ISR) that each VSI executes every time a message sent by other VSIs arrives. The second one is the periodic code that executes the particular control algorithm.

For Algorithm 1, upon reception of a message, the node identification in the message, $R X \_n o d e I D$, is used to store the incoming active power filtered measure, $\bar{p}_{r x}$. Whenever

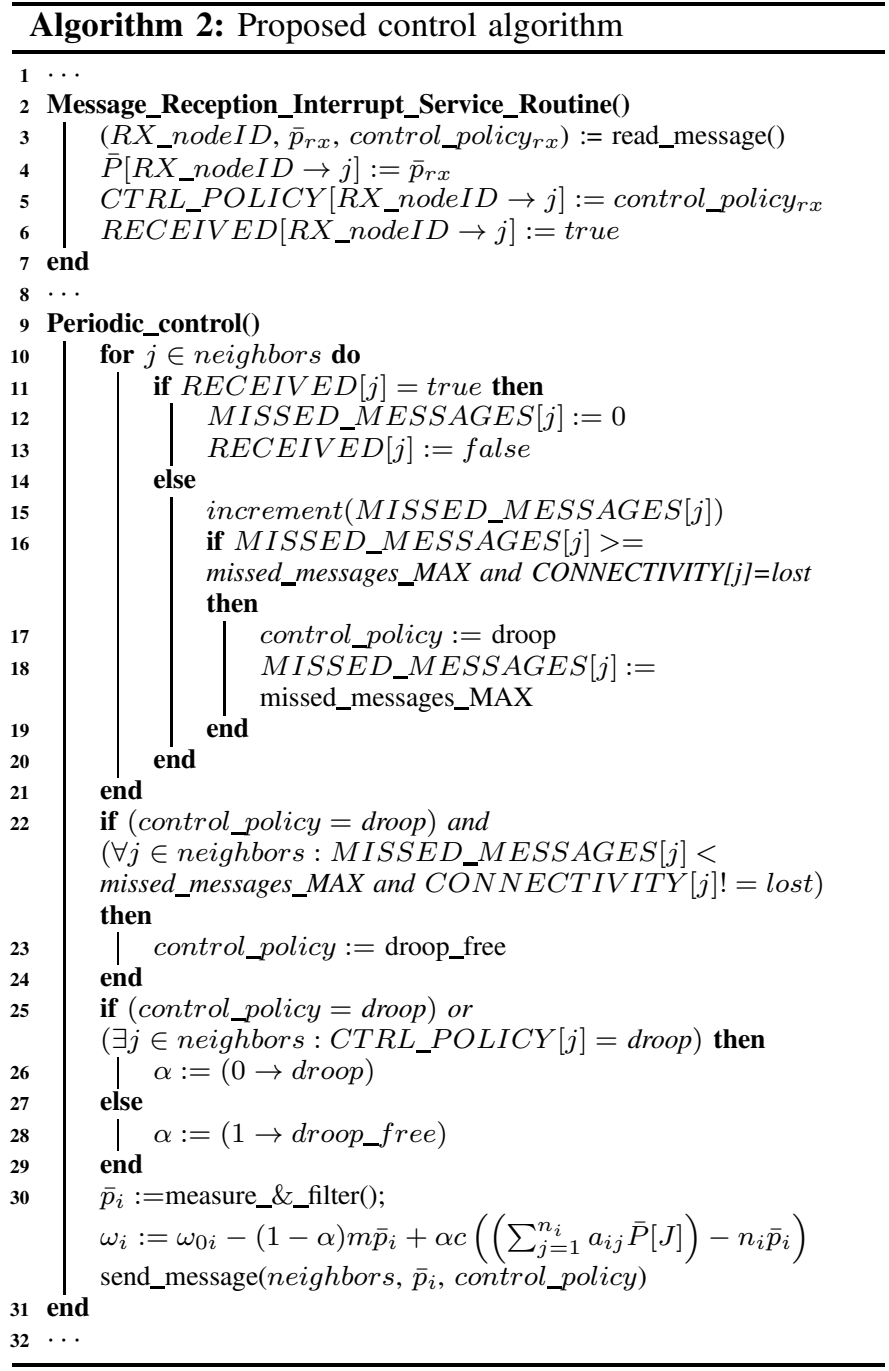

the periodic controller executes, after measuring and filtering its output power $p_{i}$, it applies the droop_free algorithm (4), and sends its filtered power to a given set of $n_{i}$ nodes, namely neighbors. The code assumes ideal communications, meaning for example that at each controller execution, all $n_{i}$ power measures for computing (6) have been received. Future work should analyze communication scenarios where messages may be lost or where synchronicity assumptions may not hold.

The proposed control strategy given in Algorithm 2 copes with the scenario where messages may be lost due to a communication partition. In the ISR, apart from storing the filtered power measure of each incoming message $\bar{p}_{r x}$, the control policy that is applying every sender (which is encoded in control_policy $y_{r x}$ with possible values being droop or droop_free) is also stored together with a true flag indicating that reception of the message of a neighbor took place (line 6).

The periodic control assumes as a default control policy the droop_free control. At each periodic execution, it first checks if there are missed messages within the expected ones specified in neighbors and takes actions accordingly (lines 10 to 21). For all received messages, a counter of missed messages for each specific neighbor node is set to zero (line 12) while the reception flag is updated to false. Otherwise, each neighbor 


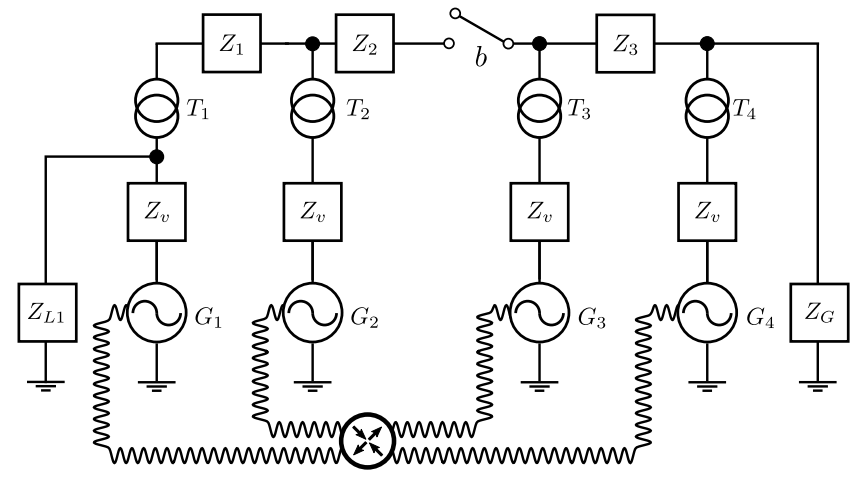

Fig. 1: Microgrid scheme

node counter of a missed messages is incremented. And if any of the counters reaches a maximum number of allowed missed messages and it implies loosing graph connectivity, then the node local control_policy is set to droop. The loss of connectivity can be detected at each VSI using two different strategies. The first one is the implementation of any existing distributed algorithm capable of detecting loss of connectivity provoking new graph partitions (e.g., [28]). The second one is to design at each VSI an event detector capable of detecting a graph partition by recognizing in the behavior of the local power and voltage the unstable dynamics that communication partitions induce. Pros and cons of implementing these two strategies for detecting partitions are not treated in this paper but left for future work.

From lines 22 to 24 the local control policy is updated to droop_free if the existing local policy was droop but all missed messages counters are below the maximum and connectivity has been recovered. In this case, an existing communication anomaly has disappeared and the standard droop-free policy is restored. Then, from line 25 to 29 , the algorithm checks if the local control policy should be droop or there are neighbors that already are executing the droop policy. If any of the two conditions hold, the node sets via the $\alpha$ flag for its own control operation the droop policy. Otherwise, it sets the flag for the droop_free policy. Finally, the control policy is applied, and the node sends its power measure and the control policy that is currently applying to a given set of $n_{i}$ neighbors nodes.

\section{EXPERIMENTAL RESULTS}

\section{A. Laboratory $M G$}

All the experiments have been performed in a laboratory MG that follows the 4-VSI scheme given in Fig. 1. It consists of a dc source (AMREL SPS-800-12) which emulates the power generation of the distributed generators $G_{1,2,3,4}$, distribution lines implemented by $R-L$ impedances and loads for power consumption (global load $Z_{G}$ and a $G_{1}$ local load with impedance $Z_{L 1}$ ). Each generator is connected to the microgrid bus through a MTL- CBI0060F12IXHF GUASCH three-phase IGBT full-bridge power inverter and an $L C$ output filter. The different control strategies of each inverter are implemented on local F28M36 digital signal processors (DSPs) from Texas
TABLE I: Nominal values of the laboratory MG components

\begin{tabular}{cll}
\hline \hline Symbol & Description & nominal value \\
\hline$v$ & Grid voltage (rms line-to-line) & $\sqrt{3} 110 \mathrm{~V}$ \\
$\omega_{0}$ & Grid frequency at no load & $2 \pi 60 \mathrm{rad} / \mathrm{s}$ \\
$Z_{1}$ & Line impedance 1 & $0.75 \Omega @ 90^{\circ}$ \\
$Z_{2}$ & Line impedance 2 & $0.30 \Omega @ 90^{\circ}$ \\
$Z_{3}$ & Line impedance 3 & $0.30 \Omega @ 90^{\circ}$ \\
$T_{1}$ & Transformer impedance & $0.62 \Omega @ 37.01^{\circ}$ \\
$T_{2}$ & Transformer impedance & $0.62 \Omega @ 37.01^{\circ}$ \\
$T_{3}$ & Transformer impedance & $1.31 \Omega @ 9.87^{\circ}$ \\
$T_{4}$ & Transformer impedance & $1.31 \Omega @ 9.87^{\circ}$ \\
$Z_{v}$ & Virtual impedance & $3.76 \Omega @ 90^{\circ}$ \\
$P_{G}$ & Nominal global load power & $1.5 \mathrm{~kW}$ \\
$P_{L 1}$ & Nominal local load power & $0.5 \mathrm{~kW}$ \\
$m$ & Gain of the frequency droop & $1 \mathrm{mrad} /(\mathrm{Ws})$ \\
$n$ & Gain of the voltage droop & $0.5 \mathrm{mV} /(\mathrm{VAr})$ \\
$c$ & Proportional gain of the droop-free & $5 \mathrm{mrad} /(\mathrm{Ws})$ \\
$\gamma$ & Filter gain of active power & 1 \\
$\omega_{c}$ & Filter cut-off frequency of active power & $2 \pi \mathrm{rad} / \mathrm{s}$ \\
\hline \hline
\end{tabular}

Instruments. The implemented strategies (only droop-free control, only frequency droop control, and the switched control strategy) are enabled with virtual impedance $Z_{v}$ plus voltage droop control (5). The latter ensures that voltages amplitudes and the values of the injected reactive power at each VSI will lie within admissible ranges. Hence, the results discussed next also show voltage amplitudes and reactive power dynamics because they permit experimentally observing their dynamics under the standard operation as well as under the partitioned scenario without or with the proposed solution.

The diagram also includes both line impedances $Z_{1,2,3}$ modeling the parasitic elements of the cables and isolation transformers $T_{1,2,3,4}$ connected at the output of each inverter. The nominal values of the MG components and control parameters are listed in Table I. The MG uses the User Datagram Protocol (UDP) over a switched Ethernet to allow communication among the four inverters. The communication network has been drawn at the bottom of Fig. 1 with a wave line. It is composed by point-to-point Ethernet cables that connects each generator to the switch (illustrated with a circle containing small arrows). The scheme includes an interruptor $b$ in the form of an electronic relay governed by a digital card. It allows the electrical partitioning of the $\mathrm{MG}$, by isolating $G_{1}-G_{2}$ from $G_{3}-G_{4}$. The communication partitioning is performed at the Ethernet switch by disabling specific communication ports. The message transmission period is set to $100 \mathrm{~ms}$ while control algorithms execute with a period of $100 \mu \mathrm{s}$. The transmission delays have not been specifically accounted for the in the implementation because they did not affect the expected MG operation in any of the analyzed cases. The output active power of all VSIs is measured using the same first order low-pass filter, whose gain $\gamma$ and cut-off frequency $\omega_{c}$ are also listed in Table I.

\section{B. Baseline Policies}

Before assessing the impact partitions, two figures are presented showing the performance of standard droop [12] and droop-free [10] policies (limited to active power sharing). The experiments have the following pattern. At times $t=0,10,20$ and $30 \mathrm{~s}$, each inverter is activated, respectively. The first 


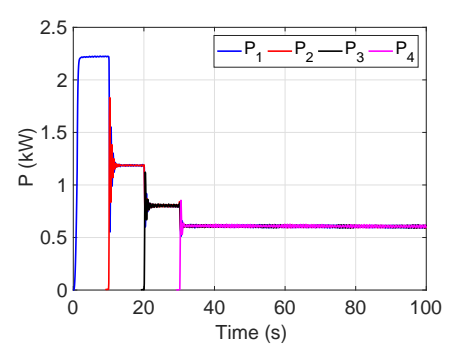

(a) Active power

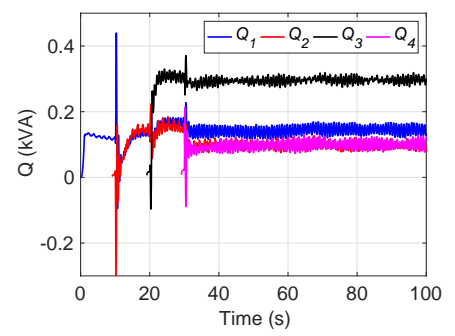

(c) Reactive power

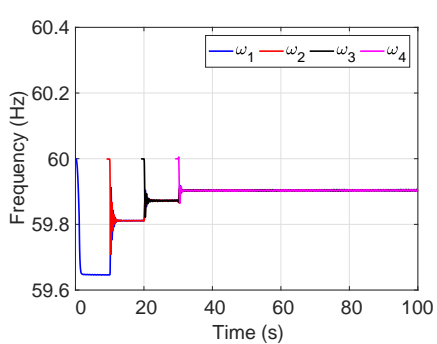

(b) Frequency

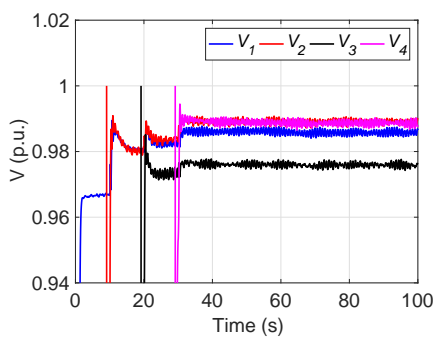

(d) Voltage amplitude

Fig. 2: System dynamics for only droop control.

generator starts and fixes a MG frequency and voltage while starting to feed both the global load and the local load, with a total power demand above than $2 \mathrm{~kW}$. The activation of the second, third and four inverters is done by means of a phaselocked loop for synchronizing them to the MG voltage phase, and also they start contributing to feed the loads.

Figures 2 and 3 show the active power delivered by each inverter (sub-figures (a)), as well as their frequencies (subfigures (b)), reactive powers (sub-figure (c)) and voltage amplitudes (sub-figure (d)). For illustrative purposes, the dynamics obtained by these two policies are assumed to be acceptable although some performance features could be improved. As it can be observed, looking at the active power sub-figures, after each connection, active power sharing is achieved, with the expected difference that transient dynamics are not the same. However, looking at the frequency sub-figures, the droop control introduces a deviation in frequency (sub-figure 2(b)) while the droop-free control keeps the frequency at the desired set-point (sub-figure 3(b)). For the sake of completeness, subfigures (c) and (d) show the dynamics of the reactive powers and voltage amplitudes for each of the tested policies. Recall that in both cases they are regulated by the voltage droop control (5).

It is worth noting that at each connection, the voltage transient dynamics (sub-figures (d) of Figures 2 and 3) exhibit a sag. These dynamics could be enhanced by a fine tuning of the control parameters and/or by a smarter soft-start of each VSI. Additionally, the frequency droop observed in subfigure (b) of Figure 2 could violate existing standards if a proper tuning of the droop control strategy is not applied. However, the paper contribution does not deal with these issues and the focus should be placed at the system dynamics whenever partitions occurs, which will occur after $t=50 \mathrm{~s}$.

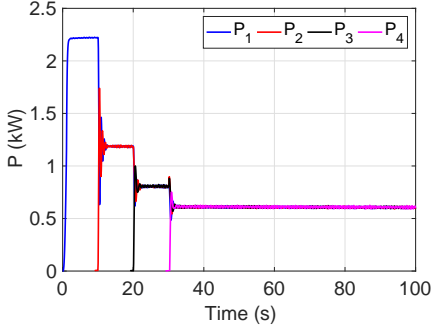

(a) Active power

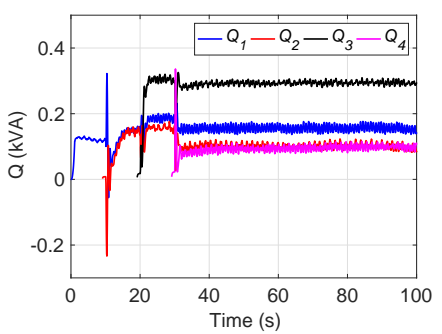

(c) Reactive power

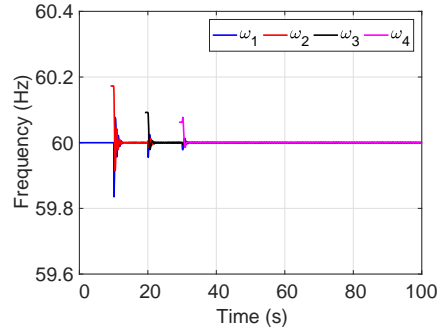

(b) Frequency

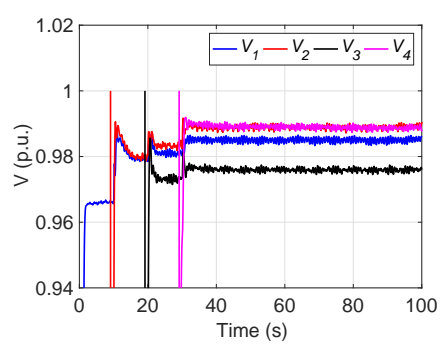

(d) Voltage amplitude
Fig. 3: System dynamics for droop-free control.

\section{Evaluation of Failures}

The next set of figures show the effect that failures have in the microgrid performance, when the MG is brought to a situation where islanded sub-networks work in parallel, both in the electrical and communication domain. In this case, the experiments have the same pattern than before but additionally, at time $t=50 \mathrm{~s}$, an electrical or a communication partition occurs, emulating a failure in a power line (opening interruptor b) or in the Ethernet switch. From the beginning of all the experiments, VSIs implement the droop-free policy (7).

When an electrical partition occurs (Fig. 4), two separate sub-MGs start working in parallel, one involving $G_{1}-G_{2}$ and feeding the local load, and the other one involving $G_{3}-G_{4}$ and feeding the global load. But both sub-MGs are governed by a "single" droop-free control algorithm, meaning that the algorithm operates without knowing that active power can not transferred between the two sub-MGs. In this case, the steadystate behavior of the active power changes compared to the normal operation of the MG (previous Fig. 3), and the injected $P_{i}$ 's reach different equilibrium points, organized in pairs and that differ among partitions. That is, $G_{1}-G_{2}$ feed the single local load thus sharing its power demand $\left(P_{1}=P_{2}\right)$, and $G_{3}-G_{4}$ feed the global load, thus sharing also its power demand $\left(P_{3}=P_{4}\right)$. If the newly reached active power values were beyond VSIs rated power, they will trip due to an overcurrent situation. Future work should consider this scenario and adopt strategies such as load shedding policies to reduce the risk of collapse.

When a communication partition occurs (Fig. 5), "two" control algorithms start acting in parallel, one involving $G_{1}-G_{2}$, and the other involving $G_{3}-G_{4}$. In fact, the algorithm executed in each VSI is the same, but they only consider for their computations the exchanged power that is available. Both generators $G_{1}$ and $G_{2}$ use $p_{1}$ and $p_{2}$ while both generators $G_{3}$ and $G_{4}$ use $p_{3}$ and $p_{4}$. Note that in this case, the four 


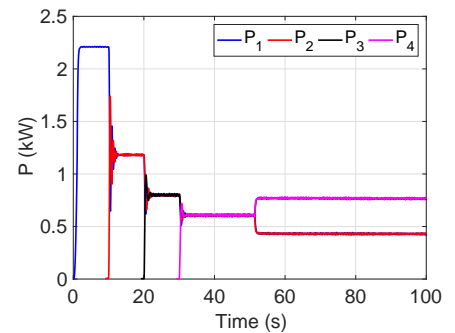

(a) Active power

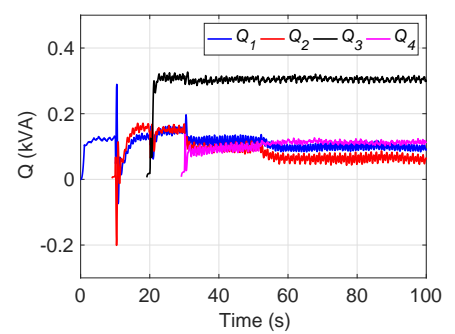

(c) Reactive power

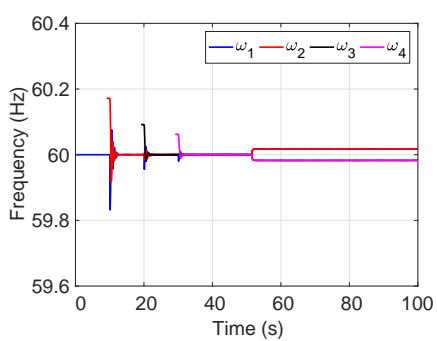

(b) Frequency

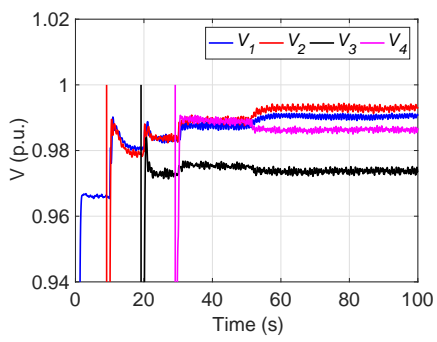

(d) Voltage amplitude

Fig. 4: System dynamics when an electrical partition leads to two sub-MGs: $G_{1}-G_{2}$ and $G_{3}-G_{4}$.

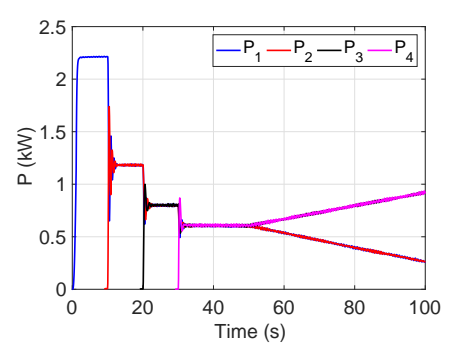

(a) Active power

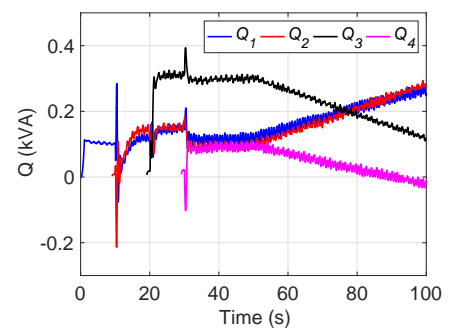

(c) Reactive power

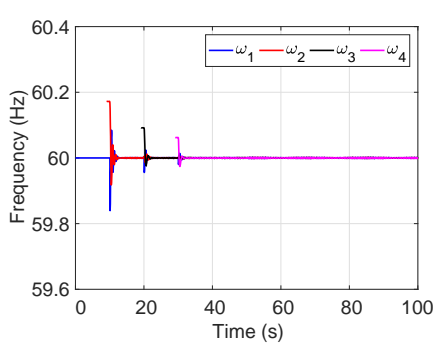

(b) Frequency

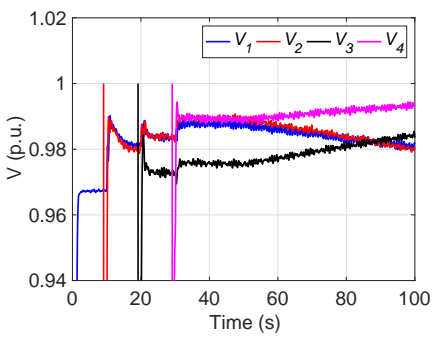

(d) Voltage amplitude

Fig. 5: System dynamics when a communication failure leads to two partitions: $G_{1}-G_{2}$ and $G_{3}-G_{4}$.

generators are still sharing the power demand of all loads. In this scenario, the injected active powers show a slow but unstable dynamics where active powers do not settle, and are grouped in pairs. In particular, $P_{1}$ and $P_{2}$ decrease and $P_{3}$ and $P_{4}$ increase. Hence, the inherent (and distinct) perturbations entering in the system (11) through the input matrices $U$ and/or $R$ such as measurement errors, effect of drifts, etc, become very important up to the point that make the dynamics unstable, that is, the MG crashes. This result corroborates the theoretically analysis presented in Section III.

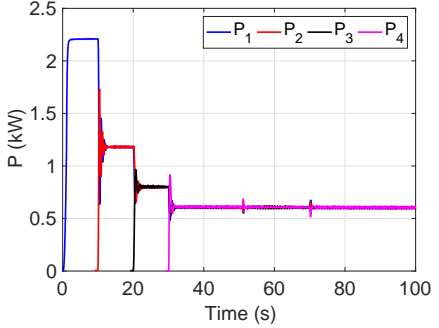

(a) Active power

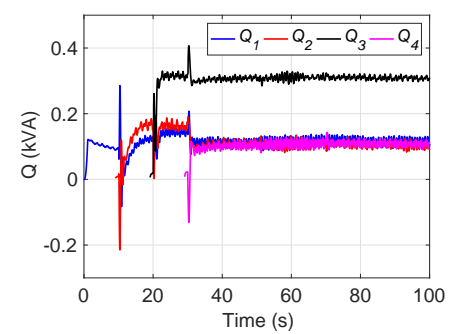

(c) Reactive power

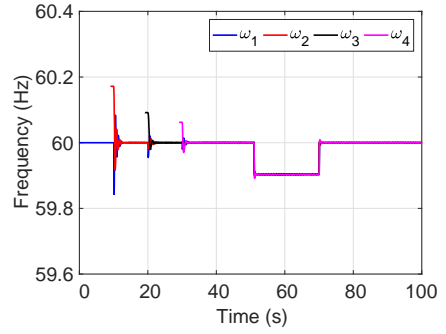

(b) Frequency

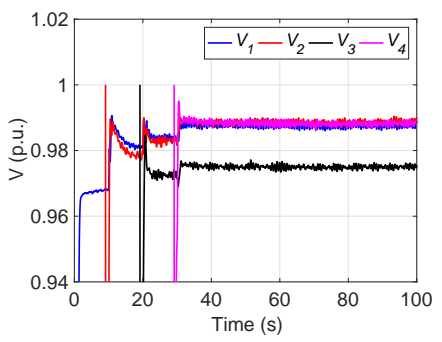

(d) Voltage amplitude
Fig. 6: System dynamics of the proposed switched control when the communication failure plotted in Fig. 5 occurs.

\section{Switched control}

In order to avoid the instability problem observed in Fig. 5, Fig. 6 shows the performance of the system when the proposed switched control strategy (33) is implemented. The experiment follows the pattern of the previous experiment, that is, at time $t=50 \mathrm{~s}$ a communication failure occurs meaning that the pair $G_{1}-G_{2}$ can not communicate with the pair $G_{3}-G_{4}$ during $20 \mathrm{~s}$, and at time $t=70 \mathrm{~s}$, communications are restored.

In this experiment, the application of the switched control is as follows. From the beginning, the droop-free control is active. When a communication failure is detected after $t=50$ $\mathrm{s}$, the frequency droop control is applied until it is detected right after $t=70 \mathrm{~s}$ that the communication network is again available and droop-free is restored. As it can be observed in the injected active powers (sub-figure 6(a)), the proposed solution has the ability of 1) avoiding the instability problem and 2) maintaining the power sharing. The pay-off is that the frequency (sub-figure 6(b)) suffers the induced and expected deviation of the frequency droop control only during the time the communication network is not available.

Finally, Fig. 7 and 8 show the case of multiple partitions. For these figures, at $t=50 \mathrm{~s}$ an electrical partition occurs implying having two separate sub-MGs, one involving $G_{1}-G_{2}$ feeding the local load, and the other one involving $G_{3}-G_{4}$ feeding the global load. At $t=70 \mathrm{~s}$, a different communication partition occurs leading to a sub-graph composed by $G_{1}-G_{4}$ that can not communicate with the sub-graph composed by $G_{2}-G_{3}$. Fig. 7 shows the effect of these partitions in the MG dynamics when droop-free control is used while Fig. 8 shows the performance of the proposed switched solution. The oscillating instability pattern shown in Fig. 7 is avoided by the switched control algorithm, as observed in Fig. 8.

The goal of the switched control strategy is avoiding the instability problem caused by the communication partition. 


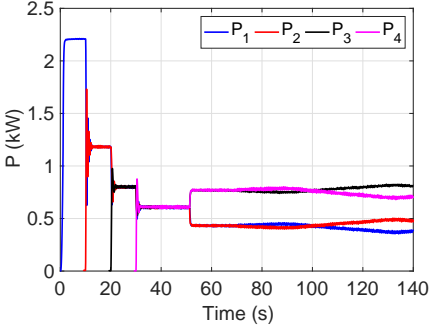

(a) Active power

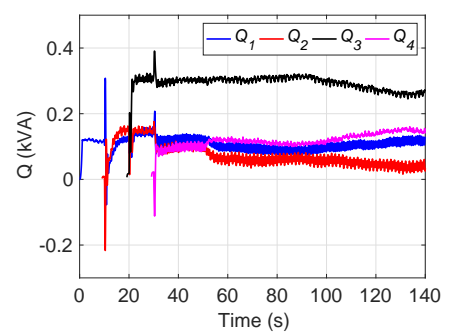

(c) Reactive power

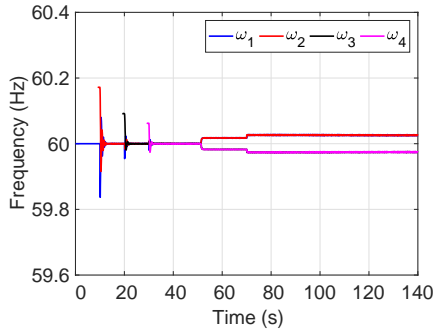

(b) Frequency

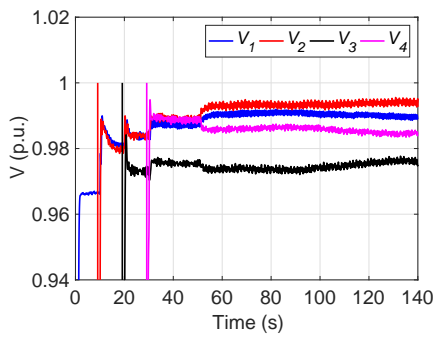

(d) Voltage amplitude

Fig. 7: System dynamics of the droop-free control when failures lead to two partitions: $G_{1}-G_{2}$ and $G_{3}-G_{4}$ in terms of electrical sub-MGs and $G_{1}-G_{4}$ and $G_{2}-G_{3}$ in terms of communication connectivity.

However, observing the frequency deviations caused by the electrical partition (sub-figure (b) in Figure 4) or by the cumulative scenario with an electrical and a communication partition (sub-figure (b) in Figure 7), future work should also consider a complementary design goal in terms of ensuring that frequency deviations should be kept within the limits mandated by existing standards.

\section{CONCLUSIONS}

This paper has analyzed the effect that communication and electrical partitions have in the performance of islanded balanced MGs governed by the droop-free algorithm. It is shown that electrical partitions generate isolated sub-MGs, and each one achieves power sharing and frequency regulation with acceptable accuracy. A communication partition results in "several" droop-free algorithms working in parallel on the same MG leading to a scenario where power sharing is lost and the system becomes unstable. A control solution has been presented to avoid the instability problem which is based on a switched control that applies droop-free control in standard operation while in the event of a communication failure it applies only the frequency droop method. Experiments in a laboratory MG illustrate the results and corroborate the goodness of the presented approach. Future work will extend the analysis to voltage amplitude and reactive power control, and it will consider richer scenarios characterized for example by time-varying and nonlinear loads, unbalanced partitions and partitions not meeting the local load demand, or communications including delays and lost of synchronicity.

\section{REFERENCES}

[1] R. H. Lasseter, "MicroGrids," in Proceedings of the 2002 IEEE Power Engineering Society Winter Meeting, pp. 305-308 vol.1., 2002.

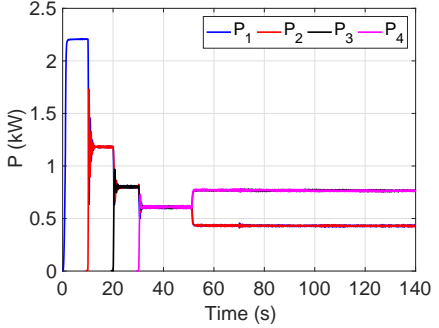

(a) Active power

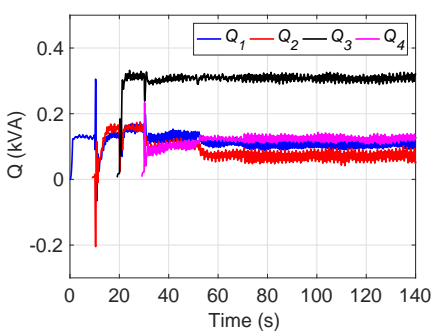

(c) Reactive power

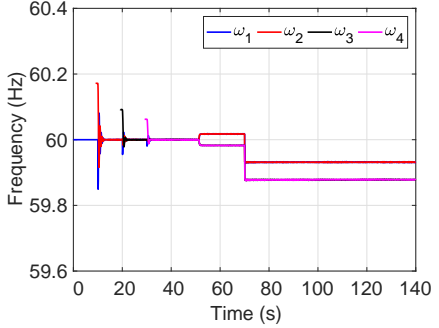

(b) Frequency

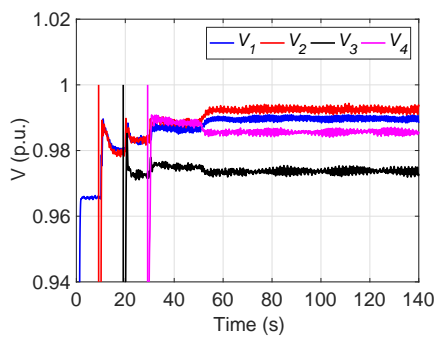

(d) Voltage amplitude
Fig. 8: System dynamics of the proposed switched control when failures lead to two partitions: $G_{1}-G_{2}$ and $G_{3}-G_{4}$ in terms of electrical sub-MGs and $G_{1}-G_{4}$ and $G_{2}-G_{3}$ in terms of communication connectivity.

[2] D. E. Olivares et al., "Trends in microgrid control," IEEE Trans. Smart Grid, vol. 5, no. 4, pp. 1905-1919, July 2014

[3] I. A. Tøndel, J. Foros, S. S. Kilskar, P. Hokstad, M. G. Jaatun, 'Interdependencies and reliability in the combined ICT and power system: An overview of current research," Applied Computing and Informatics, vol. 14, no. 1, pp. 17-27, 2018.

[4] Y. Cai, Y. Cao, Y. Li, and T. Huang, "Cascading failure analysis considering interaction between power grids and communication networks," IEEE Trans. Smart Grid, vol. 7, no. 1, pp. 1-9, 2015.

[5] A. Gholami, F. Aminifar, and M. Shahidehpour, "Front lines against the darkness: Enhancing the resilience of the electricity grid through microgrid facilities," IEEE Electrific. Mag., vol. 4, no. 1, pp. 18-24, 2016.

[6] H. F. Habib, C. R. Lashway and O. A. Mohammed, "A review of communication failure impacts on adaptive Microgrid protection schemes and the use of energy storage as a contingency," IEEE Trans. Industry Applicat., vol. 54, no. 2, pp. 1194-1207, March-April 2018.

[7] P. Danzi, M. Angjelichinoski, C. Stefanović, T. Dragicević and P. Popovski, "Software-defined microgrid control for resilience against denial-of-service attacks," IEEE Trans. Smart Grid, in press, Nov. 2018.

[8] M. Chlela, D. Mascarella, G. Joós and M. Kassouf, "Fallback control for isochronous energy storage systems in autonomous microgrids under denial-of-service cyber-attacks," IEEE Trans. Smart Grid, vol. 9, no. 5, pp. 4702-4711, Sept. 2018.

[9] S. Poudel and A. Dubey, "Critical load restoration using distributed energy resources for resilient power distribution system," IEEE Trans. Power Systems, vol. 34, no. 1, pp. 52-63, Jan. 2019.

[10] V. Nasirian, Q. Shafiee, J. M. Guerrero, F. L. Lewis and A. Davoudi, "Droop-free distributed control for AC microgrids," IEEE Trans. on Power Electronics, vol. 31, no. 2, pp. 1600-1617, Feb. 2016.

[11] J.M. Guerrero, J.C. Vasquez, J. Matas, L.G. de Vicuña, and M. Castilla, "Hierarchical control of droop-controlled AC and DC microgrids: a general approach toward standardization," IEEE Trans. on Industrial Electronics, vol. 58, no. 1, pp. 158-172, Jan. 2011.

[12] M.C. Chandorkar, D.M. Divan, and R. Adapa, "Control of parallel connected inverters in standalone AC supply systems," IEEE Trans. on Industry Applications, vol. 29, no. 1, pp. 136-143, Jan/Feb 1993.

[13] Y. Song, D. J. Hill and T. Liu, "Network-based analysis of smalldisturbance angle stability of power systems," in IEEE Transactions on Control of Network Systems, in press, 2018.

[14] C.X. Rosero, M. Velasco, P. Martí, A. Camacho, J. Miret and M. Castilla, "Analysis of consensus-based islanded microgrids subject to unexpected electrical and communication partitions," in IEEE Transactions on Smart Grid, in press, 2018. 
[15] S. Nourollah and G. B. Gharehpetian, "Coordinated load shedding strategy to restore voltage and frequency of microgrid to secure region," IEEE Trans. Smart Grid, in press, 2019.

[16] F. Dörfler and F. Bullo, "Kron reduction of graphs with applications to electrical networks," IEEE Trans. on Circuits and Systems I: Regular Papers, vol. 1, no. 60, pp. 150-163, 2013.

[17] P. Kundur, Power System Stability and Control, McGraw-Hill, 1994.

[18] X. Guo, Z. Lu, B. Wang, X. Sun, L. Wang and J. M. Guerrero, "Dynamic phasors-based modeling and stability analysis of droop-controlled inverters for microgrid applications," in IEEE Transactions on Smart Grid, vol. 5, no. 6, pp. 2980-2987, Nov. 2014.

[19] J. Matas, M. Castilla, L. García de Vicuña, J. Miret and J.C. Vasquez, "Virtual impedance loop for droop-controlled single-phase parallel inverters using a second-order general-integrator scheme", IEEE Trans. Power Electronics, vol. 25, no. 12, pp. 2993 - 3002, Dec. 2010.

[20] F. Dörfler, J. W. Simpson-Porco and F. Bullo, "Electrical networks and algebraic graph theory: models, properties, and applications," in Proceedings of the IEEE, vol. 106, no. 5, pp. 977-1005, May 2018.

[21] M.C. Pulcherio, A.A. Renjit, M.S. Illindala, A.S. Khalsa, J.H. Eto, D.A. Klapp and R.H. Lasseter, "Evaluation of control methods to prevent collapse of a mixed-source microgrid," in IEEE Transactions on Industry Applications, vol. 52, no. 6, pp. 4566-4576, Nov.-Dec. 2016.

[22] M. Khederzadeh and A. Beiranvand, "Identification and prevention of cascading failures in autonomous microgrid," in IEEE Systems Journal, vol. 12, no. 1, pp. 308-315, March 2018.

[23] S. Banerjee and A.Roy, Anindya. Linear Algebra and Matrix Analysis for Statistics. Texts in Statistical Science (1st ed.), Chapman and Hall/CRC, 2014.

[24] C. Godsil and G. Royle. Algebraic Graph Theory. Springer, 2001.

[25] M. Andreasson, D. V. Dimarogonas, H. Sandberg and K. H. Johansson, "Distributed control of networked dynamical systems: Static feedback, integral action and consensus", in IEEE Trans. on Automatic Control, vol. 59 , no. 7 , pp.1750 -1764, 2014.

[26] D. Liberzon, Switching in Systems and Control, Birkhäuser Basel, 2003.

[27] A.R. Meenakshi and C. Rajian, "On a product of positive semidefinite matrices," in Linear Algebra and its Applications, vol. 295, no. 1-3, pp. 3-6, 1999.

[28] C. Jain, P. Flick, T. Pan, O. Green and S. Aluru, ”An adaptive parallel algorithm for computing connected components," IEEE Trans. Parallel Distrib. Syst., vol. 28, no. 9, pp. 2428-2439, Sept. 2017.

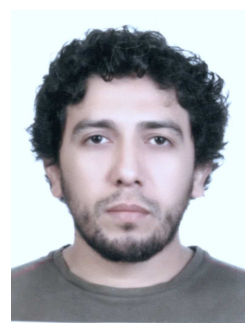

Carlos Xavier Rosero received the B.Sc. degree in automotive electronics engineering from the Army Polytechnic School, Sangolqui, Ecuador in 2008, and the M.Sc. degree in automatic control and robotics from the Technical University of Catalonia (UPC), Barcelona-Spain in 2015. He is currently working toward the Ph.D. degree in automatic control, robotics and vision from UPC. His research interests are centered on real-time control systems and microgrids with emphasis on control strategies.

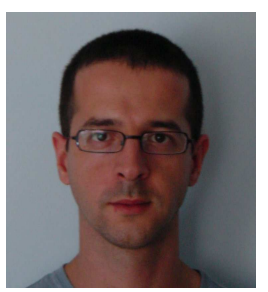

Manel Velasco graduated in maritime engineering in 1999 and received the $\mathrm{PhD}$ degree in automatic control in 2006, both from the Technical University of Catalonia, Barcelona, Spain. Since 2002, he has been an assistant professor in the Department of Automatic Control at the Technical University of Catalonia. His research interests include artificial intelligence, real-time control systems, and collaborative control systems, especially on redundant controllers and multiple controllers with self-interacting systems.

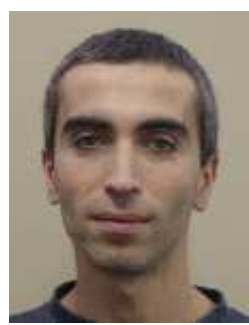

Pau Martí (M'02) received the degree in computer science and the $\mathrm{PhD}$ degree in automatic control from the Technical University of Catalonia, Barcelona, Spain, in 1996 and 2002, respectively. During 2003 he held a research fellow appointment in the Computer Science Department at the University of California at Santa Cruz, US. From 1996 to 2013, he has been an assistant professor in the Department of Automatic Control at the Technical University of Catalonia. Since 2013 he has been an Associate Professor in the Department of Automatic Control at the Technical University of Catalonia. His research interests include embedded and networked control systems, nonlinear control and microgrids.

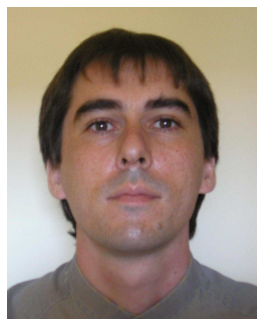

Antonio Camacho received the B.S. degree in chemical engineering, the M.S. degree in automation and industrial electronics, and the Ph.D. degree in electronic engineering, from the Technical University of Catalonia, Barcelona, Spain in 2000, 2009 and 2015 respectively. His research interests include networked and embedded control systems, industrial informatics, and power electronics.

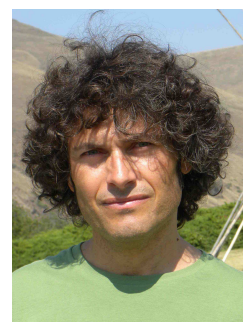

Jaume Miret (M'98) received the B.S. degree in telecommunications, M.S. degree in electronics, and $\mathrm{Ph} . \mathrm{D}$. degree in electronics from the Universitat Politecnica de Catalunya, Barcelona, Spain, in 1992, 1999, and 2005, respectively. From 1993 to 2011, he was an Assistant Professor in the Department of Electronic Engineering, Universitat Politecnica de Catalunya, Spain. Since 2011 he has been an Associate Professor in the Universitat Politecnica de Catalunya, where he teaches courses on digital design and circuit theory. His research interests include dc-to-ac converters, active power filters, and digital control.

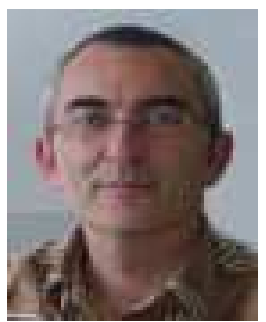

Miguel Castilla received the B.S., M.S. and Ph.D. degrees in telecommunication engineering from the Technical University of Catalonia, Barcelona, Spain, in 1988, 1995, and 1998, respectively. Since 2002, he has been an Associate Professor in the Department of Electronic Engineering, Technical University of Catalonia, where he teaches courses on analog circuits and power electronics. His research interests are in the areas of power electronics, nonlinear control, and renewable energy systems. 